\title{
Modelo de requerimiento hídrico en un distrito de riego en México: incorporando escenarios de cambio climático
}

\author{
Model of water requirement in an irrigation district in Mexico: under climate change scenarios
}

\begin{abstract}
Edgar Omar Ruiz-Del-Angel ${ }^{1 *}$, Rocío Del Carmen Vargas Castilleja ${ }^{2}$, Julio Cesar Rolón Aguilar ${ }^{2}$, Carlos Alberto Chávez García ${ }^{1}$

1 Facultad de Ingeniería, Universidad Autónoma de Querétaro, C.U Cerro de las Campanas, 76010 Querétaro, México.

2 División de Estudios de Posgrado e Investigación, Facultad de Ingeniería "Arturo Narro Siller", Universidad Autónoma de Tamaulipas, Ave. Universidad y Blvd. Adolfo López Mateos, Centro Universitario Tampico-Madero, C.P. 89109 Tampico, Tamaulipas, Tamaulipas, México.
\end{abstract}

\section{RESUMEN}

El conocimiento de la cantidad de agua que requiere un cultivo es uno de los factores fundamentales para lograr un plan de riego eficiente. En la actualidad se han desarrollado paquetes tecnológicos que permiten estimar los volúmenes de requerimiento de agua tomando en cuenta las condiciones climatológicas y del suelo. En México la superficie regable está conformada por 86 Distritos de Riego (DR) y 40,000 Unidades de Riego. La presente investigación tuvo como objetivo modelar los requerimientos de agua para el DR 002, de Mante en Tamaulipas, México, considerando registros históricos de una serie de años agrícolas, un clima base y escenarios de cambio climático. Para la modelación se utilizó el software CROPWAT versión 8.0; el cultivo analizado fue la caña de azúcar; el periodo histórico comprendió los ciclos agrícolas 2007-2014; la climatología de referencia fue la de Hijmans et al. (2005) extraída de la base de datos WorldClim 1950-2000; los Modelos de Circulación General fueron el GFDL-CM3 y HADGEM2-ES para las Trayectorias de Concentración Representativas (RCP, en inglés) 4.5 y 8.5 $\mathrm{W} / \mathrm{m}^{2}$. Con los resultados se realizó una comparativa entre el volumen requerido y los volúmenes que en realidad se emplean, encontrándose que en la mayoría de los casos el volumen requerido es mayor que el realmente empleado, incluso mayor que el concesionado $\left(165.7 \mathrm{Mmm}^{3}\right)$ para aprovechamiento.

Palabras clave: Distrito de riego, evapotranspiración, precipitación, CROPWAT, caña de azúcar.

\section{ABSTRACT}

The knowledge of the quantity of water that a crop requires is one of the fundamental factors to achieve an efficient irrigation plan. At present, technological packages are available which allow the estimation of the volumes of water requirement considering the climatological and soil conditions. In Mexico, the irrigable area is made up of 86 Irrigation Districts (DR) and 40,000 Irrigation Units. The objective of this research was to model the water requirements for DR 002, from Mante in Tamaulipas, Mexico, considering the historical records of a series of agricultural years, a reference climatolo- gy and climate change scenarios. CROPWAT 8.0 was used for the modeling; the crop analyzed was sugarcane; the historical period comprised the agricultural cycles 2007-2014; the reference climatology was that of Hijmans et al., extracted from the WorldClim database 1950-2000; The General Circulation Models were the GFDL-CM3 and HADGEM2-ES for the Representative Concentration Trajectories (RCP) 4.5 and 8.5 . With the results, we compared the volume required and the volumes used, finding that in most cases the volume required is greater than actually used, even greater than the concession (165.7 $\mathrm{Mmm}^{3}$ ) for utilization.

Key words: Irrigation district, evapotranspiration, precipitation, CROPWAT, sugarcane.

\section{INTRODUCCIÓN}

La agricultura es una actividad primaria fundamental para el desarrollo de cualquier civilización y llevarla a cabo no es una tarea nada sencilla, es el ecosistema de suministro de alimentos más grande y más importante del mundo, cubriendo el $38 \%$ de la superficie del planeta (Hutton et al., 2018). En esta actividad intervienen una gran cantidad de factores que afectan directa o indirectamente la producción de cualquier cultivo, como es el caso del agua. En Europa el sector agrícola consume aproximadamente el $51.4 \%$ del total del agua dulce extraída, mientras que en EE.UU le corresponde el $31 \%$ del volumen de agua y se espera que el impacto de cambio climático (ICC) aumente la presión sobre el recurso hídrico a nivel mundial de un $70 \%$ a un $90 \%$ hacia el año 2050 (loannis et al., 2018). Actualmente, el fenómeno de cambio climático es reconocido por la comunidad científica y comprender en qué medida y en que magnitud los sistemas se verán afectados por este, es fundamental para el diseño de medidas de mitigación y de adaptación (Paredes et al., 2018). EI ICC afecta directamente la escorrentía y por consiguiente la disponibilidad de agua, también afecta la demanda de agua (Döll, 2002). En combinación con el crecimiento de la población, es probable que el ICC juegue un papel significativo en la disponibilidad de agua en el futuro (White et al., 2014). Si la temperatura global del planeta aumenta en promedio $2^{\circ} \mathrm{C}$, los modelos actuales sugieren que es casi inevitable 
que una quinta parte de la población mundial sufra escasez de agua, por lo que una medida necesaria, será aumentar la productividad de los recursos hídricos existentes (Banerjee et al., 2016). En el caso del riego, optimizar el agua disponible es muy importante (Cavero et al., 2000). Para la optimización del riego se requieren predicciones del uso de agua de los cultivos, la producción de los cultivos entre otros factores (Zhang et al., 2018). En México la superficie territorial con fines para la agricultura es aproximadamente de 30.2 millones de hectáreas de las cuales sólo el $18 \%$ son de regadío y el resto tiene régimen de temporal. Las 6.5 millones de hectáreas que cuentan con infraestructura de riego se encuentran divididas en 86 distritos de riego (DR) y alrededor de 40 mil unidades de riego (UR) de esta manera México ocupa el séptimo lugar mundial en cuanto a superficie con infraestructura de riego (CONAGUA, 2017). Los DR y las UR son elementos muy importantes tanto para la seguridad alimentaria como para la economía de México, debido no solo a la producción de alimentos sino también a la generación de empleos, incremento al ingreso y mejoramiento de las condiciones de vida del medio rural (Iñiguez et al., 2015) En el presente trabajo se realizó una modelación mediante el software CROPWAT versión 8.0 para los ciclos agrícolas 2007-2008, 2008-2009, 2009-2010, 2010-2011, 2011-2012, 2012-2013 у 2013-2014 para el DR 002 Mante, Tamaulipas en México. Los volúmenes obtenidos mediante la modelación se compararon con los volúmenes programados y realizados en el DR. Posteriormente, se generaron 4 modelaciones considerando el ICC con el aumento de temperatura promedio del planeta a $2{ }^{\circ} \mathrm{C}$, para los Modelos de Circulación General GFDL-CM3 y HADGEM2-ES y para las Trayectorias de Concentración Representativas (RCP) 4.5 y $8.5 \mathrm{~W} / \mathrm{m}^{2}$, también se generó una modelación usando la climatología base de WorldClim, propuesta por Hijmans et al. (2005) para la serie 1950-2000, los 5 modelos anteriores se realizaron considerando eficiencias en la conducción de 50\%, $60 \%$ y $70 \%$.

\section{MATERIAL Y MÉTODOS Área de estudio}

El DR 002 Mante está ubicado en el municipio El Mante, en el Estado de Tamaulipas, México (Figura 1), cuenta con una superficie total de 19,165.3 Ha, de las cuales la superficie de riego es alrededor de 15,250 $\mathrm{Ha}$; esta superficie puede variar de un año a otro. En este DR se cultiva principalmente la caña de azúcar, siendo ocupada el $99.5 \%$ de la superficie para el desarrollo de este cultivo, donde el $0.5 \%$ restante corresponde a cítricos y pastizales.

\section{Modelación}

Para realizar la modelación se utilizó el programa CROPWAT versión 8.0, una herramienta desarrollada por científicos de la Organización de las Naciones Unidas para la Alimentación y la Agricultura (FAO, por sus siglas en inglés), es una aplicación práctica que auxilia en el cálculo de las necesidades del cultivo a partir de información climatológica y propia de los cultivos (Arteaga et al., 2011). Entre las funciones

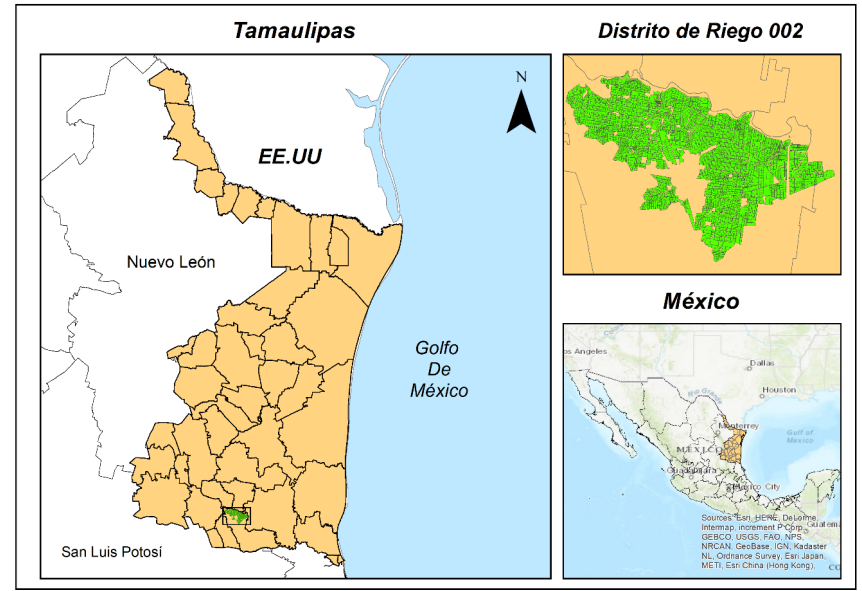

Figura 1. Localización del Distrito de Riego 002, Mante, Tamaulipas, México. Figure 1. Location of Irrigation District 002, Mante, Tamaulipas, Mexico.

principales del programa, destacan calcular la evapotranspiración de referencia, los requerimientos de agua del cultivo, así como los requerimientos de riego, también permite realizar programas de riego para diferentes escenarios (Stancalie et al., 2010). Para realizar la modelación en CROPWAT se requiere como insumos, las temperaturas máximas y mínimas, las precipitaciones medias y las características de los cultivos, mismos que se describirán en los siguientes apartados. Con la finalidad de contrastar los volúmenes de requerimiento obtenidos mediante el software y los volúmenes que se programaron y que en realidad se otorgaron, se tomaron los insumos acordes al periodo analizado.

\section{Evapotranspiración}

La modelación requiere las temperaturas máximas y mínimas para calcular la evapotranspiración de referencia. Para obtener dichas temperaturas primero se buscaron las estaciones meteorológicas del Servicio Meteorológico $\mathrm{Na}$ cional (SMN) que se encontraban dentro y fuera del DR 002; se tomaron ocho estaciones (Figura 2). La información de temperatura se extrajo de manera mensual para el periodo 2007-2014, posteriormente para cada ciclo, se realizó una interpolación mediante un Sistema de Información Geográfica (SIG). A partir de esta interpolación, se obtuvieron isotermas para todos los meses, tomándose finalmente la temperatura media máxima o mínima de cada mes como el promedio de las isotermas que cruzan a lo largo de todo el DR. Para calcular la evapotranspiración de referencia mediante el software, se colocaron los datos de la estación climatológica elegida, debido a que se trabajó con varias estaciones, se optó por elegir un punto de referencia, las coordenadas y altitud de este, fueron los que se introdujeron al programa, cuyo punto fue a $22.773383^{\circ}$ latitud norte y $98.966029^{\circ}$ longitud oeste con una altitud de 60 metros sobre el nivel del mar. El paso final para integrar las temperaturas a CROPWAT, es tomar en cuenta el año agrícola. En México el año agrícola comprende desde el 01 de octubre de un año hasta el 30 de septiembre del siguiente año, i.e. para el año agrícola 2007-2008 se to- 


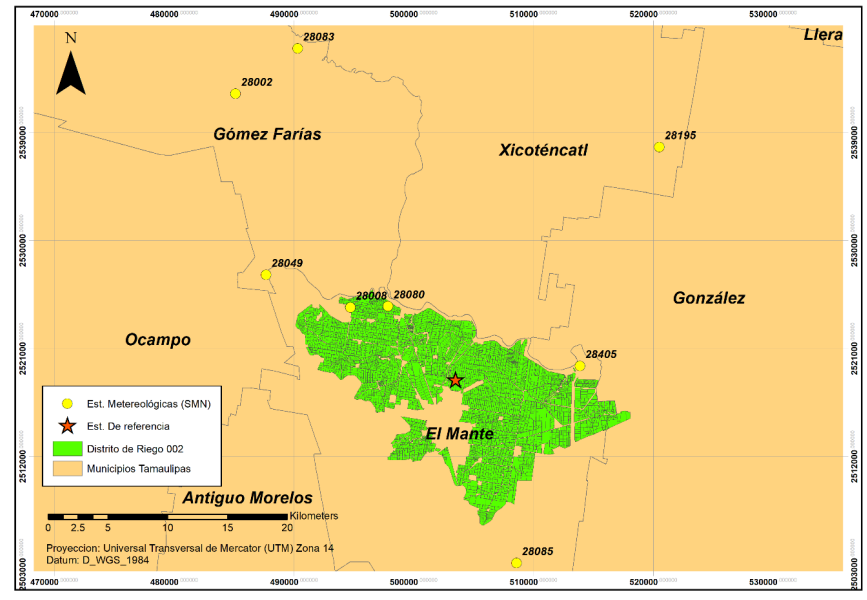

Figura 2. Distrito de Riego 002 y estaciones meteorológicas del Servicio Meteorológico Nacional.

Figure 2. Irrigation District 002 and meteorological stations of the National Meteorological Service.

man las temperaturas de los meses de octubre, noviembre y diciembre del 2007, y los meses restantes se tomaron del año 2008, de la misma manera se realizó para todos los años agrícolas comprendidos dentro del periodo de análisis. La evapotranspiración se calcula mediante el método de Penman - Monteith (FAO, 2018) como se muestra en la ecuación 1.

$$
E T_{o}=\frac{0.408 \Delta\left(R_{n}-G\right)+\gamma \frac{900}{T+273} u_{2}\left(e_{s}-e_{a}\right)}{\Delta+\gamma\left(1+0.34 u_{2}\right)} \quad \text { EC. } 1
$$

Donde $E T_{o}$ es la evapotranspiración de referencia en $\left(\mathrm{mm} \mathrm{dia}{ }^{-1}\right) ; R_{n}$ es la radiación neta en la superficie del cultivo $\left(\mathrm{Mjm}^{-2} \mathrm{dia}^{-1}\right) ; R_{a}$ es la radiación extraterrestre $\left(\mathrm{mm} \mathrm{dia}^{-1}\right) ; G$ es el flujo de calor del suelo en $\left(\mathrm{Mjm}^{-2} \mathrm{dia}^{-1}\right)$; Tes la temperatura media del aire a $2 \mathrm{~m}$ de altura $\left({ }^{\circ} \mathrm{C}\right) ; u_{2}$ es la velocidad del viento a $2 \mathrm{~m}$ de altura $\left(\mathrm{m} \mathrm{s}^{-1}\right) ; e_{\mathrm{s}}$ es la presión de vapor a saturación $(k P a) ; e_{a}$ es la presión real de vapor $(k P a) ; e_{s}-e_{a}$ se toma como el déficit de presión de vapor $(\mathrm{kPa}) ; \Delta$ es la pendiente de la curva de presión de vapor en $\left(\mathrm{kPa}{ }^{\circ} \mathrm{C}^{-1}\right)$; y finalmente es la constante psicrometrica $\left(\mathrm{KPa}^{\circ} \mathrm{C}^{-1}\right)$.

\section{Precipitación}

Para obtener la precipitación media del DR 002, se utilizaron las ocho estaciones meteorológicas que se mencionan en el apartado anterior, primero se obtuvieron las láminas acumuladas de forma mensual para cada año del periodo 2007-2014. Posteriormente, mediante el método de polígonos de Thiessen (Aparicio, 1989), se determinaron las precipitaciones medias mensuales para el DR. Una vez obtenida la precipitación media mensual, CROPWAT estima la precipitación efectiva, es decir, la porción de la lluvia que es aprovechada por las plantas, para el presente trabajo se usó la ecuación 2 que sugiere la $\mathrm{FAO}$

$$
\begin{cases}P_{E f}=0.6 P-10 & P \geq 70 \mathrm{~mm} \\ P_{E f}=0.8 P-24 & P>70 \mathrm{~mm}\end{cases}
$$

donde $P_{E f}$ es la precipitación efectiva y $P$ es la precipitación media mensual, ambas en (mm). Para introducir las lá- minas de precipitación al software se consideró la referencia del año agrícola, tomando los meses de octubre, noviembre y diciembre de un año y los meses de enero a septiembre del siguiente año.

\section{Caña de azúcar}

Para la modelación se tomó como único cultivo la caña de azúcar, debido a que la superficie total de riego del DR en estudio representa un $99.5 \%$. De la superficie total, se estima según información propia del DR, que cada año se renueva alrededor de un $25 \%$ de superficie de caña, mientras que el otro $75 \%$ es considerado como soca o caña con dos o más ciclos de corte, tomando como referencia lo anterior para cada uno de los años modelados. Un $25 \%$ del total de la superficie destinada a caña de azúcar se manejó como planta o caña virgen y el otro $75 \%$ como soca y resoca. Para el módulo de cultivo en CROPWAT, se emplearon valores estandarizados que corresponden a la caña de azúcar ( $F$ A O, 2018). En la tabla 1 se muestran los valores característicos para la soca y resoca, que son casi los mismos para la planta, variando solamente la duración de las etapas de crecimiento siendo para esta última de 50, 70, 220 y 140 días. Las anteriores etapas suman un total de 480 días. Dentro de los valores estándar se manejó la altura máxima del cultivo como $3 \mathrm{~m}$. Adicionalmente, en este módulo correspondiente al cultivo, se introduce la fecha de siembra y la duración de las etapas de desarrollo, a partir de esta información el software estima la fecha de cosecha. Con base en la literatura, la fecha de siembra en un cultivo perene se considera cuando a la planta le comienzan a brotar las hojas, después que se ha cosechado. Lo anterior aplica para el caso de la caña soca y resoca, por lo que en la presente investigación se establecieron diferentes

Tabla 1. Valores de cultivo en la modalidad Soca y Resoca.

Table 1. Crop values in the Soca and Resoca modality.

\begin{tabular}{lccccc}
\hline Etapa de crecimiento & Inicial & Desarrollo & Media & Final & Total \\
\hline Duración (días) & 30 & 50 & 180 & 100 & 360 \\
$\begin{array}{l}\text { Coeficiente de cultivo } \\
\text { (Kc) }\end{array}$ & 0.4 & & 1.25 & 0.75 & \\
$\begin{array}{l}\text { Profundidad Radicular } \\
\text { (m) }\end{array}$ & 0.5 & & 1.6 & 1.6 & \\
$\begin{array}{l}\text { Agotamiento crítico } \\
\text { (Fracción) }\end{array}$ & 0.6 & & 0.6 & 0.6 & \\
\hline $\begin{array}{l}\text { Factor de respuesta } \\
\text { al rendimiento (Ky) }\end{array}$ & 1.1 & 1.15 & 1.18 & 1.2 & \\
\hline
\end{tabular}

Tabla 2. Fechas de siembra para las modelaciones de la soca y resoca. Table 2. Seeding dates for the soca and resoca modeling.

\begin{tabular}{lcc}
\hline Año Agrícola & Fecha de Siembra & Fecha de cosecha \\
\hline $2007-2008$ & $20-$ Dic & $14-$ Dic \\
\hline $2008-2009$ & $01-$ Ene & $26-$ Dic \\
\hline $2009-2010$ & $15-$ Ene & $09-$ Ene \\
\hline $2010-2011$ & $05-$ Nov & $19-$ Ene \\
\hline $2011-2012$ & $05-$ Feb & $30-$ Ene \\
$2012-2013$ & $15-$ Feb & $09-$ Feb \\
\hline $2013-2014$ & $01-$ Mar & $23-$ Feb \\
\hline
\end{tabular}


fechas de siembra, para lograr una secuencia (Tabla 2), ya que se generó un modelo de requerimiento independiente para cada año agrícola. Para la superficie correspondiente a la planta o caña virgen, la fecha de siembra se consideró al 18 de julio, esto para todos los casos.

\section{Suelo}

Para integrar el módulo relativo al suelo, se requieren los valores correspondientes a la capacidad de campo del suelo (CC), siendo el agua excedente, después que ha sido saturado el suelo y el proceso de drenaje ha terminado (Lambers et al., 2008). En este caso se consideró $0.34 \mathrm{~cm}^{3} / \mathrm{cm}^{3}$. El punto de marchitamiento permanente (PMP), es el contenido de agua que queda una vez que la planta se ha marchitado completamente (Müller, 1964); para fines del modelo se tomó $0.19 \mathrm{~cm}^{3} / \mathrm{cm}^{3}$. La diferencia entre las dos propiedades anteriores fue de $0.15 \mathrm{~cm}^{3} / \mathrm{cm}^{3}$, lo que para fines del modelo es 150 $\mathrm{mm} / \mathrm{m}$. Por último, se requiere la tasa máxima de infiltración, conocida como la conductividad hidráulica a saturación (Ks), que es la velocidad con la que el flujo de agua viaja a través del suelo ya saturado (Forsythe, 1985); para la modelación se tomó como $36 \mathrm{~mm} / d i ́ a$. Las propiedades anteriormente descritas varían con respecto a la textura del suelo, en el caso del DR 002 el suelo es de tipo franco arcilloso y los valores que fueron usados en la modelación son valores estándar para este tipo de suelo (Zataráin, Fuentes and Vauclin, 2012). También se utilizaron valores obtenidos del manual de la FAO (2018). La profundidad radicular se tomó de la Tabla 1, donde el agotamiento inicial de humedad del suelo se mantuvo en cero para todas las modelaciones.

\section{Eficiencia}

En el recorrido que hace el agua desde la fuente de suministro ya sea presa o pozo, hasta la parcela donde se llevará a cabo el riego, sufre de pérdidas y estas dependen directamente de la eficiencia del sistema de conducción. La eficiencia de conducción muestra el volumen relativo de agua que se pierde en los canales (Pedroza, 2014), es expresada en porcentaje y determina el volumen bruto de agua que se está consumiendo. Para el caso de las modelaciones realizadas en este estudio y contemplando un contraste al finalizar las mismas, las eficiencias que se consideraron fueron las registradas en el DR en cada uno de los años agrícolas analizados cuya media es de $56.77 \%$. En el DR aproximadamente el $85 \%$ de los usuarios realizan el riego con prácticas empíricas (Vargas et al., 2017).

\section{Climatología de referencia}

Para realizar una modelación y obtener el volumen de agua que será requerido por los cultivos en un futuro, se utilizan las normales de precipitación y temperatura. Las normales son medias que se calculan a partir de un periodo uniforme y suficientemente largo, abarca por lo menos tres décadas consecutivas y tienen como finalidad ser una referencia que contribuyen a realizar predicciones (WMO, 2007).
En este estudio se utilizaron las normales climatológicas de la base WorldClim Global Climate Data para la serie 1950-2000, la cual se encuentra interpolada a una resolución espacial de $926 \mathrm{~m}$ por $926 \mathrm{~m}$, considerando el efecto topográfico (Hijmans et al., 2005). Para el caso de la precipitación, se extrajeron los valores de cada píxel mediante un SIG para posteriormente aplicar polígonos de Thiessen y obtener la precipitación media mensual para el área del DR. En el caso de las temperaturas, solo se extrajeron los valores para cada píxel y después se obtuvo un promedio aritmético de todos los valores, de esta manera se integraron los módulos de evapotranspiración y lluvia en el modelo para obtener los volúmenes de requerimiento de agua para un año referencia.

\section{Impacto de cambio climático}

Considerar el impacto de cambio climático permite desarrollar mecanismos y herramientas para mitigar y adaptarse a las nuevas condiciones climatológicas (Vargas et al., 2017). Para el desarrollo del presente estudio se realizaron modelaciones considerando dos Modelos de Circulación General Océano-Atmósfera (AOGCM's, por sus siglas en inglés) que se desprenden del Quinto Informe de Evaluación del Panel Intergubernamental de Cambio Climático (AR5) (IPCC, 2014), el GFDL-CM3 y HADGEM2-ES; para 2 forzamientos radiativos o vías de concentración representativas, el RCP $4.5 \mathrm{~W} / \mathrm{m}^{2}$ (emisiones bajas) y RCP $8.5 \mathrm{~W} / \mathrm{m}^{2}$ (emisiones altas) (Fernández Eguiarte et al., 2014). Finalmente, se utilizaron sólo las anomalías correspondientes, en el supuesto de que la temperatura global se incrementara $2^{\circ} \mathrm{C}$ y que se estima se estaría presentando a partir del año 2051 (INECC, 2017).

Las anomalías que representan el impacto del cambio climático se sumaron o restaron a la climatología de referencia, según fuera el caso, obteniéndose las precipitaciones y temperaturas bajo los efectos de los escenarios de cambio climático. En el caso de la precipitación, se extrajeron los valores de cada píxel, mediante un SIG, posteriormente se aplicaron polígonos de Thiessen y se obtuvo la precipitación media ponderada. En el caso de la temperatura máxima y mínima, se extrajeron los valores de todos los pixeles del DR para después realizar un promedio aritmético. Para todas las modelaciones con ICC la fecha de siembra se tomó al 20 de diciembre para el caso de la soca y resoca; en el caso de la planta se mantuvo como al 18 de julio, y la superficie de siembra se consideró como 15,475 ha, siendo la mayor su-

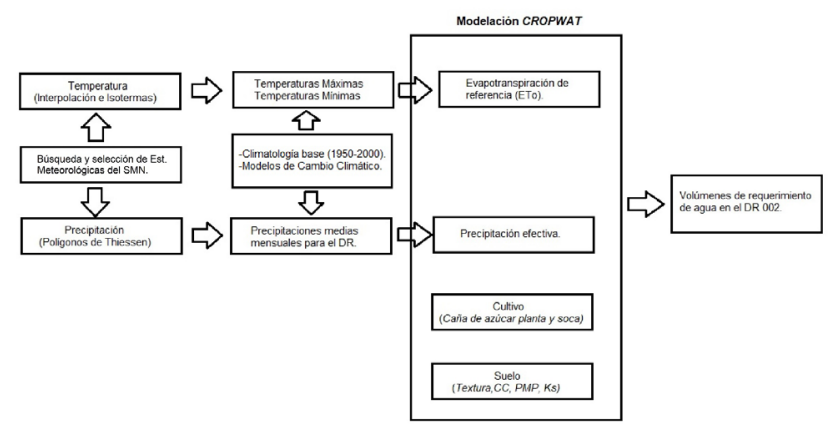

Figura 3. Esquema de la metodología empleada. Figure 3. Scheme of the methodology used. 
perficie sembrada dentro del análisis histórico que se realizó. Se manejaron eficiencias de 50, 60 y $70 \%$.

\section{Esquema}

Para finalizar esta sección, se muestra de manera esquemática la metodología que se siguió para obtener los volúmenes de requerimiento de agua para el DR 002 (Figura 3).

\section{RESULTADOS Y DISCUSIÓN Evapotranspiración}

Los valores más altos de evapotranspiración, cercanos a los $6 \mathrm{~mm} /$ día fueron para los meses de abril, mayo, junio, julio y agosto, mientras que los más bajos se presentaron en los meses de diciembre y enero con valores a $2 \mathrm{~mm} /$ día. Las evapotranspiraciones considerando la climatología de referencia, presentan similitud con el análisis histórico; los valores máximos son para los meses de abril, mayo, junio, julio y agosto con valores cercanos a $5.5 \mathrm{~mm} /$ día, mientras que los valores mínimos fueron para diciembre y enero con valores de $2.75 \mathrm{~mm} /$ día. Para las evapotranspiraciones obtenidas considerando el ICC se aprecia un aumento para los meses de diciembre y enero, con valores cercanos a los $3 \mathrm{~mm} /$ día. Los valores máximos fueron abril, mayo y junio con valores

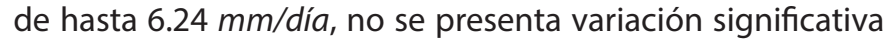
entre los modelos con ICC.

\section{Precipitación}

La precipitación media ponderada en el caso del análisis histórico se obtuvo a partir de la información de las estaciones meteorológicas del SMN utilizando el método de polígonos de Thiessen. Primero de manera mensual para cada año y posteriormente se integraron los años agrícolas que se introdujeron al software para generar la lámina de lluvia efectiva. Los meses más lluviosos son junio, julio, agosto y septiembre con precipitaciones efectivas hasta de $270 \mathrm{~mm}$, mientras que los más secos son noviembre, diciembre, enero y febrero donde en ocasiones no se presentaron precipitaciones efectivas. En el caso de la climatología de referencia y la impactada con cambio climático, los meses más lluviosos son los mismos que los del análisis histórico; para el caso del ICC se muestra una reducción en las láminas de precipitación en los meses de verano con precipitaciones efectivas de hasta de $150 \mathrm{~mm}$, mientras que en los meses de invierno se muestra un pequeño incremento, registrando precipitaciones efectivas pequeñas.

\section{Volúmenes de requerimiento de agua obtenidos en CROPWAT}

El modelo estima de manera diaria y mensual el volumen neto de agua que requiere un cultivo, el cálculo lo hace a partir de la diferencia que existe entre la precipitación efectiva y la evapotranspiración del cultivo. Para obtener el volumen de requerimiento de agua total de cada año agrícola, se elaboró una plantilla de cálculo donde a partir de la lámina neta (LN), resultado de la modelación y la eficiencia de riego (ER), se calculó una lámina de riego bruta (LB). Con
Tabla 3. Volúmenes de requerimiento para la soca y resoca en el año agrícola 2013-2014.

Table 3. Volumes of requirements for soca and resoca in the 2013-2014 agricultural year.

\begin{tabular}{lcccccc}
\hline Mes & LN (mm) & ER (\%) & LB (mm) & Sup.(Ha) & VN & VB \\
\hline Ene & 64.5 & 57.79 & 111.61 & $9,937.5$ & 6.410 & 11.091 \\
Feb & 48.9 & 57.79 & 84.62 & $9,937.5$ & 4.859 & 8.409 \\
\hline Mar & 30.2 & 57.79 & 52.26 & $9,937.5$ & 3.001 & 5.193 \\
\hline Abr & 73.3 & 57.79 & 126.84 & $9,937.5$ & 7.284 & 12.605 \\
\hline May & 127.2 & 57.79 & 220.11 & $9,937.5$ & 12.641 & 21.873 \\
\hline Jun & 18.6 & 57.79 & 32.19 & $9,937.5$ & 1.848 & 3.198 \\
\hline Jul & 75.8 & 57.79 & 131.16 & $9,937.5$ & 7.533 & 13.034 \\
\hline Ago & 153.3 & 57.79 & 265.27 & $9,937.5$ & 15.234 & 26.361 \\
\hline Sep & 0 & 57.79 & 0 & $9,937.5$ & 0 & 0 \\
\hline Oct & 112.1 & 57.79 & 193.98 & $9,937.5$ & 11.140 & 19.277 \\
\hline Nov & 85.8 & 57.79 & 148.47 & $9,937.5$ & 8.526 & 14.754 \\
\hline Dic & 66.9 & 57.79 & 115.76 & $9,937.5$ & 6.648 & 11.504 \\
\hline Total & & & & & 85.125 & 147.300 \\
\hline
\end{tabular}

las dos láminas de agua y la superficie de riego (Sup.), se realizó el cálculo del volumen de agua neto (VN) y el volumen de agua bruto (VB) en millones de metros cúbicos de forma mensual, para finalmente obtener el acumulado anual. Como ejemplo de la plantilla, para el cálculo del volumen total neto y bruto para la soca en el año agrícola 2013-2014 se muestra la Tabla 3. Al volumen obtenido de la soca se le suma el volumen requerido por la porción que le corresponde a la caña virgen o planta. En una primera fase del análisis histórico se obtuvieron los volúmenes de requerimiento totales para cada uno de los años agrícolas, posteriormente y considerando las condiciones operativas en el DR, se realizaron algunos ajustes a los volúmenes obtenidos, lo anterior debido a que un periodo de días antes y después de la fecha tentativa de corte, no se debe de realizar riego alguno, aunque el cultivo lo requiera. Antes de la cosecha, el ingenio azucarero establece un periodo de 45 días de no riego y una vez que se efectúa el corte de la caña no se riega hasta que se hayan realizado los trabajos correspondientes, como es el caso del rebote y los trabajos de subsuelo. Tomando en cuenta lo anterior, se omitieron los requerimientos de agua para 2 meses antes y 2 meses después de la fecha de corte. Los volúmenes con y sin ajustes se pueden observar en las gráficas de las Figuras 4 y 5 . En las gráficas anteriores, se observa que los volúmenes programados no presentan variación significativa a lo largo de los años agrícolas, mientras que en los volúmenes consumidos se presentan algunas diferencias notables, por ejemplo, en el año agrícola 2008-2009 se consumieron 177.5 $\mathrm{Mmm}^{3}$, mientras que para el periodo 2009-2010 se consumió un volumen bruto de $85.9 \mathrm{Mmm}^{3}$.

\section{Volúmenes del análisis histórico}

Se llevó a cabo la comparación con los volúmenes programados y los volúmenes realizados (Figura 4). Se puede observar que para algunos años agrícolas los volúmenes de CROPWAT se aproximan a los programados, como es el caso 


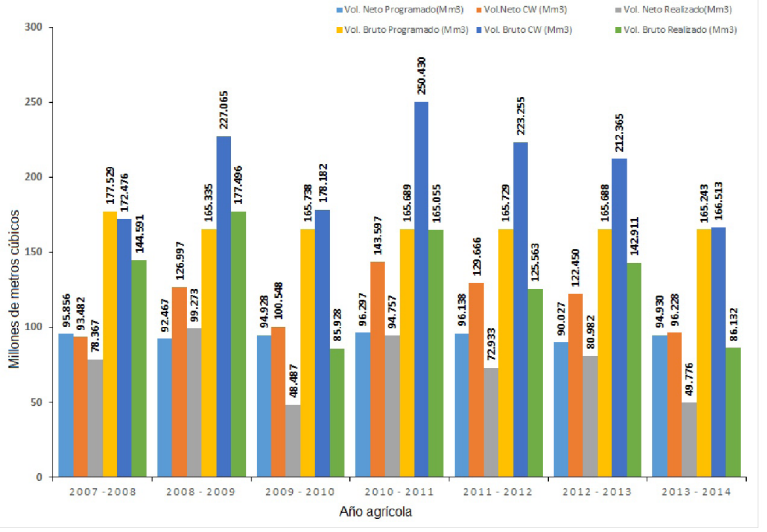

Figura 4. Comparación de volúmenes para el Distrito de Riego 002 (análisis histórico).

Figure 4. Comparison of volumes for Irrigation District 002 (historical analysis).

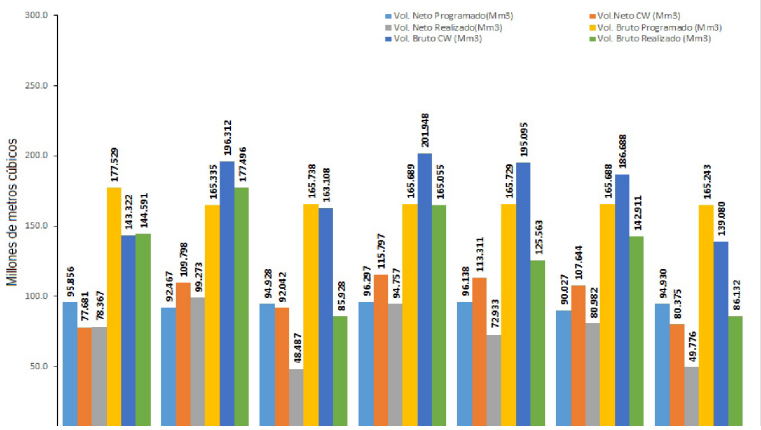

Figura 5. Comparación de volúmenes para el Distrito de Riego 002, aplicando ajustes a los resultados de CROPWAT (análisis histórico).

Figure 5. Comparison of volumes for Irrigation District 002, applying adjustments to CROPWAT results (historical analysis).

de 2007-2008 y 2013-2014, mientras que, para todos los demás años, los volúmenes provenientes de las modelaciones son mayores, lo que indica que los requerimientos de agua de la caña de azúcar en el DR son mayores a los que realmente se estiman. Estos volúmenes no contienen ningún tipo de ajuste, se presentan con base en los resultados del modelo. Cuando se realiza el ajuste correspondiente y al quitar los volúmenes de 2 meses antes y 2 meses después de la fecha de corte, los volúmenes de requerimiento de CROPWAT se acercan más a los volúmenes programados y realizados, como se observa en la Figura 5. Una mejor percepción del contraste en los volúmenes se muestra en las Figuras 6 y 7 , donde se pueden observar los volúmenes programados y realizados contra los volúmenes obtenidos en la modelación. Cuando los porcentajes son negativos, significa que el volumen obtenido en CROPWAT resultó mayor que el volumen con el cual se está comparando, de esta manera como se observa en la Figura 6, para el año agrícola 2007-2008 el volumen programado fue mayor en un $2.93 \%$ que el volumen obtenido en CROPWAT; sin embargo, en el mismo año el volumen

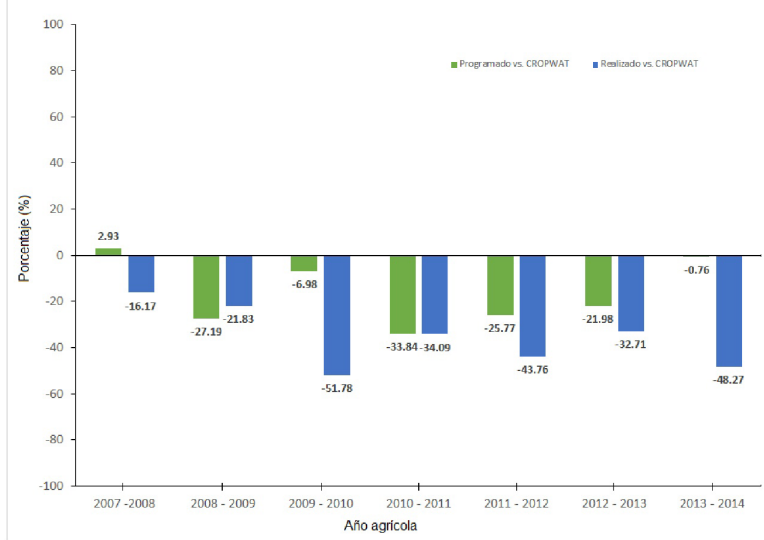

Figura 6. Comparación de volúmenes en porcentaje (análisis histórico). Figure 6. Comparison of volumes as percentage (historical analysis).

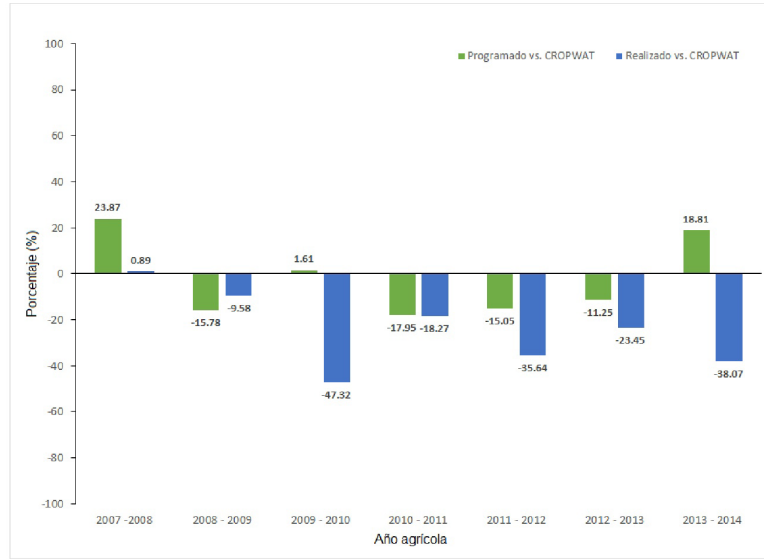

Figura 7. Comparación de volúmenes en porcentaje, aplicando ajustes (análisis histórico).

Figure 7. Comparison of volumes as percentage, applying adjustments (historical analysis).

realizado fue menor que el volumen de la modelación en un $16.17 \%$.

\section{Volúmenes para la climatología de referencia e ICC}

Al realizar la modelación para la climatología de referencia y la impactada por escenarios de cambio climático considerando un incremento global de la temperatura de $2^{\circ} \mathrm{C}$, se obtuvo que el volumen de requerimiento de agua neto para Hijmans et al. (2005) es de 107-977 $\mathrm{Mmm}^{3}$ el cual está por debajo de la media obtenida del análisis histórico (2007-2014) sin aplicar ajustes, el cual fue de $116.138 \mathrm{Mmm}^{3}$ (Figura 8). Mientras que, considerando el ICC, los volúmenes netos de requerimiento aumentan a123 y $124 \mathrm{Mmm}^{3}$, que se traduce en un $15 \%$ más con respecto a la climatología de referencia y un $6.5 \%$ más, de la media obtenida en el periodo histórico analizado. Para el caso de los volúmenes brutos, que están en función de la eficiencia de la conducción, y como los escenarios futuros no la consideran, se realizó la modelación para tres eficiencias; 50\%, 60\% y 70\% (Figura 9). Para la climatología de referencia los volúmenes de requerimiento fueron 


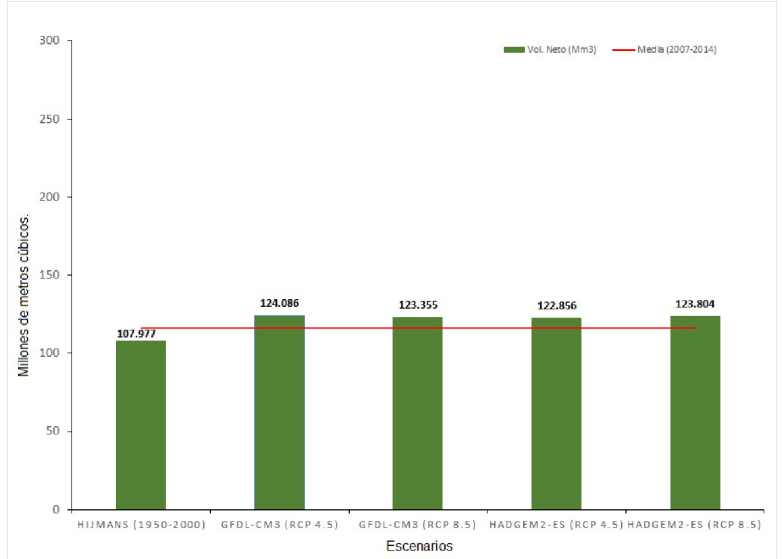

Figura 8. Volúmenes netos con Impacto de Cambio Climático para el Distrito de Riego 002.

Figure 8. Net volumes with Climatic Change Impact for Irrigation District 002 .

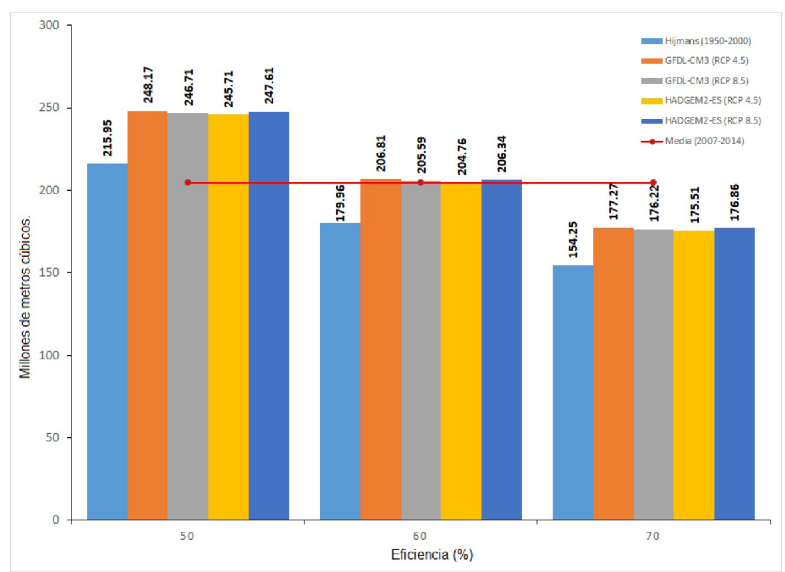

Figura 9. Volúmenes de agua totales en bruto para el Distrito de Riego 002, considerando Impacto de Cambio Climático.

Figure 9. Raw total water volumes for Irrigation District 002, considering Climatic Change Impact.

215, 179 y $154 \mathrm{Mmm}^{3}$ respectivamente. Los volúmenes brutos con ICC se compararon con la media de los volúmenes brutos obtenidos mediante el análisis histórico, que fue de 204.327 $\mathrm{Mmm}^{3}$. Como se observa, para una eficiencia de 50\% los volúmenes considerando el ICC son del orden de 245 a $248 \mathrm{Mmm}^{3}$, esto es aproximadamente $21.5 \%$ más de la media del análisis histórico. Para la eficiencia de $60 \%$ los volúmenes con ICC estuvieron en el orden de 204 a $207 \mathrm{Mmm}^{3}$ esto es prácticamente la media del análisis histórico. Finalmente, para la eficiencia de $70 \%$ los volúmenes de requerimiento fueron del orden de 175 a $177 \mathrm{Mmm}^{3}$ aproximadamente un $13 \%$ menos de la media del análisis histórico.

\section{CONCLUSIONES}

A partir de las modelaciones realizadas en CROPWAT para diferentes años agrícolas y para escenarios con ICC, fue posible estimar los volúmenes de requerimiento de agua de la caña de azúcar para el DR 002. En el caso del análisis histórico se pudo realizar una comparación entre los volúmenes modelados y los realmente empleados (en promedio), encontrándose que en algunos años agrícolas el cultivo requirió un volumen de agua mayor al que verdaderamente se empleó, como es el caso de los años 2008-2009, 2010-2011, 20112012 y 2012-2013 donde la variación de volúmenes es mayor (de 22\% a 52\%.) La variación entre los volúmenes de requerimiento del cultivo y los que realmente se emplean atienden principalmente a dos factores, el primero relacionado con la actitud particular de cada usuario con respecto al riego, esto determina la prontitud con la que efectúa. El segundo factor, es el programa de corte de la caña, que en muchas ocasiones entra en conflicto con el programa de riego, por lo que este último se suspende. Tomando en cuenta estos dos factores, es explicable porqué en algunos años agrícolas hay una variación evidente entre los volúmenes del modelo, los volúmenes programados y los realizados. Todos los resultados de las modelaciones tanto para el análisis histórico como para la climatología de referencia y con ICC, los volúmenes brutos de requerimiento de agua para la caña de azúcar sin ajustes estuvieron por arriba del volumen que la Comisión Nacional del Agua (CONAGUA) tiene concesionado al DR 002, que es de $165.7 \mathrm{Mmm}^{3}$. En el análisis histórico, incluso aplicando los ajustes correspondientes de los meses antes y después de la fecha de corte, de los siete años o ciclos agrícolas analizados, en cuatro de ellos, el volumen de requerimiento es mayor que el volumen concesionado. Una productividad óptima es otorgar al cultivo la cantidad de agua necesaria, por ello el proceso de estimación del volumen de requerimiento y la elaboración de un programa de riego seguido a cabalidad, es fundamental para obtener buenos resultados en los rendimientos del cultivo, coadyuvando al aprovechamiento del recurso agua.

\section{AGRADECIMIENTOS}

Se agradece la colaboración del Gerente Operativo de la Comisión de Cuenca Río Guayalejo-Tamesí, el Ing. Miguel Moreno Martínez, por sus aportes fundamentales para la construcción del modelo. De igual manera, se reconoce el apoyo brindado al director de Ingenieros Sin Fronteras México, A.C., Dr. Gerardo Sánchez Torres Esqueda, así como a la División de Estudios de Posgrado e Investigación de la Facultad de Ingeniería, a través de su jefe de la División y líder del Cuerpo Académico Consolidado UAT-CA-29, Dr. Julio Cesar Rolón Aguilar.

\section{REFERENCIAS}

Aparicio, F. 1989. Fundamentos de Hidrología de Superfície. 1ra ed. Limusa. México.

Arteaga, R., Ángeles, V. y Vázquez, M. A. 2011. Programa cropwat para planeación y manejo del recurso hídrico. Revista Mexicana de Ciencias Agrícolas. 2: 179-195.

Banerjee, S., Chatterjee, S., Sarkar, S. y Jena, S. 2016. Projecting Future Crop Evapotranspiration and Irrigation Requirement of Potato in Lower Gangetic Plains of India using the CROPWAT 8.0 Model. Potato Research. Potato Research. 59(4): 313-327. doi: 10.1007/s11540-016-9327-7. 
Cavero, J., Farre, I., Debaeke, P. y Faci, J. 2000. Simulation of Maize Yield Under Water Stress with EPICphase and CROPWAT in a Semiarid Climate. Agronomy Journal. 92(March): 679-690.

CONAGUA. 2017. Estadisticas Del Agua en México. Comisión Nacional del Agua. Ciudad de Mexico.

Döll, P. 2002. Impact of Climate Change and Variability on Irrigation Requirements: A Global Perspective. Climatic Change. 54: 269-293. doi: 10.1023/A:1016124032231.

F A O, Evapotranspiración del cultivo. [Consultado 20 mayo 2018] 2018. Disponible en: http://www.fao.org/3/a-x0490s. pdf.

Fernández, A., Zavala, J., Romero, R., Conde, A. y Trejo, R. 2014. Actualización de los escenarions de cambio climático para estudios de impactos, vulnerabilidad y adaptación en México y Centroamérica. doi: 04-2011-120915512800-203.

Forsythe, W. 1985. Física de Suelos. 1ra ed. IICA. San Jose Costa Rica.

Hijmans, R. J., Cameron, S. E., Parra, J. L., Jones, P. G. y Jarvis, A. 2005. Very high resolution interpolated climate surfaces for global land areas. International Journal of Climatology. 25(15): 1965-1978. doi: 10.1002/joc.1276.

Hutton, C. W., Adger, W. N., Hanson, S. E. y Salehin, M. 2018. Prospects for Agriculture Under Climate Change ans Soil Salinisation. En: Ecosystem Services for Well-Being in Deltas. p. 593. doi: 10.1007/978-3-319-71093-8.

INECC. 2017. Estimación de rangos de incertidumbre en las fechas para alcanzar los valores de incremento en la temperatura promedio global $1.0,1.5$ y $2.0^{\circ} \mathrm{C}$ y las implicaciones para la República Mexicana como producto de la Sexta Comunicación Nacional de México ante la Convención Marco de las Naciones Unidas sobre el Cambio Climático.Ciudad de México. México.

Iñiguez, M., Ojeda, W. y Díaz, C. 2015. La infraestructura hidroagrícola ante escenarios del cambio climático TT Hydro-Agricultural Infrastructure under Climate Change Scenarios. Tecnología y ciencias del agua. 6(5): 89-101.

loannis D. T., Zoidou M., Georgios D.G. y Sylaios G. K. 2018. Impact of Irrigation Technologies and Strategies on Cotton Water Footprint Using AquaCrop and CROPWAT Models. Environmental Processes. doi: https://doi.org/10.1007/ s40710-018-0289-4.

IPCC. 2014. Informe de síntesis, Cambio climático 2014: Informe de síntesis. Contribución de los Grupos de Trabajo I, II y III al Quinto Informe de Evaluación del Grupo Intergubernamental de Expertos sobre el Cambio Climático [Equipo principal de redacción, R.K. Pachauri y L.A. Meyer (eds.)].IPCC,Ginebra, Suiza.

Lambers, H., Chapin, F. S. y Pons, T. L. 2008. Plant Physiological Ecology. 2da ed. Springer. New York. EE.UU. doi: 10.1007/9780-387-78341-3.
Luo, X., Xia, J. y Yang, H. 2015. Modeling water requirements of major crops and their responses to climate change in the North China Plain. Environmental Earth Sciences, Springer Berlin Heidelberg. 74(4):3531-3541. doi: 10.1007/s12665015-4400-0.

Müller, L. E. 1964. Manual de laboratorio de fisiología vegetal. Instituto interamericano de ciencias agricolas de la O.E.A. Turrialba

Paredes, J., Gómez, M. A., Mastachi, C.A., Diaz, C., Becerril, R., Martinez, H. y Khalidou, M.B. 2018. Impacts of Climate Change on the Irrigation Districts of the Rio Bravo Basin. Water. 1: 1-20. doi: 10.3390/w10030258.

Pedroza, E. y Hinojosa, G. 2014. Manejo y distribución del agua en distritos de riego. Breve introducción didáctica. Instituto Mexicano de Tecnología del Agua. Jiutepec, Morelos, México.

Stancalie, G., Marica, A. y Toulios, L. 2010. Using earth observation data and CROPWAT model to estimate the actual crop evapotranspiration. Physics and Chemistry of the Earth. Elsevier Ltd. 35(1-2): 25-30. doi: 10.1016/j.pce.2010.03.013.

Vargas, R., Rolón, J., Pichardo, R. y Treviño, J. 2017. Programa de Acción Climática de Tamaulipas. En: La Gobernanza Climática en México:Aportes para la consolidación estructural de la participación ciudadana en la política climática nacional. $\mathrm{O}$. Gutierrez-Fuentes., D.O Jerez-Ramírez., P. Ligregni-Aguilera., L. López-Morales., R.F Sánchez-Lara. y V. Vidal-Aguirre (ed.), pp 253-270. Universidad Autónoma de México. Ciudad de México. México.

Vargas, R., Rolón, J., Treviño, J. y Ruiz, E. 2017. Disponibilidad del Recurso Hídrico Superficial. En: El recurso hídrico en Tamaulipas: la cuenca del Río Guayalejo Tamesí. G. ArcosEspinosa., D.M.E González-Turrubiates. y E. Martínez-Cano (ed.), pp 147-185. Palibrio. Tampico, Tamaulipas, México.

White, C. J., Tanton, T. W. y Rycroft, D. W. 2014. The Impact of Climate Change on the Water Resources of the Amu Darya Basin in Central Asia. Water Resources Management. 28(15): 5267-5281. doi: 10.1007/s11269-014-0716-x.

WMO. 2007. Función de las normales climatológicas en un clima cambiante.

Zataráin, F., Fuentes, C. y Vauclin, M. 2012. Capítulo 5. Variabilidad Espacial de los Suelos y el Riego. En: Riego por Gravedad. C.Fuentes. y L. Rendón (ed.) pp 238-262. Universidad Autónoma de Querétaro. Querétaro, México.

Zhang, Y., Hansen, N., Trout, T., Nielsen, D. y Paustian, K. 2018. Modeling Deficit Irrigation of Maize with the DayCent Model. Agronomy Journal. 118:1-11. doi: 10.2134/ agronj2017.10.0585. 